\title{
Analysis of a simulated microarray dataset: Comparison of methods for data normalisation and detection of differential expression (Open Access publication)
}

Michael Watson ${ }^{\mathrm{a} *}$, Mónica PÉREZ-ALEGRE ${ }^{\mathrm{b}}$, Michael Denis BARON $^{\mathrm{c}}$, Céline DELMAS $^{\mathrm{d}}$, Peter Dovč ${ }^{\mathrm{e}}$, Mylène Duval ${ }^{\mathrm{d}}$, Jean-Louis FoulLEY ${ }^{\mathrm{f}}$, Juan José GARRIDO-PAVó ${ }^{\mathrm{b}}$, Ina HULSEGGE $^{\mathrm{g}}$, Florence JAFFRÉZIC ${ }^{\mathrm{f}}$, Ángeles JIMÉNEZ-MARÍN ${ }^{\mathrm{b}}$, Miha LAVRIČ ${ }^{2}$, Kim-Anh LÊ CAO ${ }^{\mathrm{h}}$, Guillemette MAROT ${ }^{\mathrm{f}}$, Daphné MOUZAKI ${ }^{\mathrm{h}}$, Marco H. PoOL ${ }^{\mathrm{c}}$, Christèle RoberT-GranIÉ ${ }^{\mathrm{d}}$, Magali SAN CRISTOBAL ${ }^{\mathrm{d}}$, Gwenola TOSSER-KLOPP ${ }^{\mathrm{d}}$, David WADDINGTON $^{\mathrm{h}}$, Dirk-Jan DE KONING ${ }^{\mathrm{h}}$

\author{
${ }^{a}$ Institute for Animal Health, Compton, UK (IAH_C) \\ ${ }^{\mathrm{b}}$ University of Cordoba, Cordoba, Spain (CDB) \\ ${ }^{\mathrm{c}}$ Institute for Animal Health, Pirbright, UK (IAH_P) \\ ${ }^{\mathrm{d}}$ INRA, Castanet-Tolosan, France (INRA_T) \\ ${ }^{\mathrm{e}}$ University of Ljubljana, Slovenia (SLN) \\ ${ }^{\mathrm{f}}$ INRA, Jouy-en-Josas, France (INRA_J) \\ g Animal Sciences Group Wageningen UR, Lelystad, NL (IDL) \\ ${ }^{\mathrm{h}}$ Roslin Institute, Roslin, UK (ROSLIN)
}

(Received 10 May 2007; accepted 10 July 2007)

\begin{abstract}
Microarrays allow researchers to measure the expression of thousands of genes in a single experiment. Before statistical comparisons can be made, the data must be assessed for quality and normalisation procedures must be applied, of which many have been proposed. Methods of comparing the normalised data are also abundant, and no clear consensus has yet been reached. The purpose of this paper was to compare those methods used by the EADGENE network on a very noisy simulated data set. With the a priori knowledge of which genes are differentially expressed, it is possible to compare the success of each approach quantitatively. Use of an intensity-dependent normalisation procedure was common, as was correction for
\end{abstract}

*Corresponding author: michael.watson@bbsrc.ac.uk Institute for Animal Health Informatic groups, Compton Laboratory, Compton RG20 7 NN Newbury Bershive, UK. 
multiple testing. Most variety in performance resulted from differing approaches to data quality and the use of different statistical tests. Very few of the methods used any kind of background correction. A number of approaches achieved a success rate of $95 \%$ or above, with relatively small numbers of false positives and negatives. Applying stringent spot selection criteria and elimination of data did not improve the false positive rate and greatly increased the false negative rate. However, most approaches performed well, and it is encouraging that widely available techniques can achieve such good results on a very noisy data set.

gene expression / two colour microarray / simulation / statistical analysis

\section{INTRODUCTION}

Microarrays have become a standard tool for the exploration of global gene expression changes at the cellular level, allowing researchers to measure the expression of thousands of genes in a single experiment [16]. The hypothesis underlying the approach is that the measured intensity for each gene on the array is proportional to its relative expression. Thus, biologically relevant differences, changes and patterns may be elucidated by applying statistical methods to compare different biological states for each gene. However, before comparisons can be made, a number of normalisation steps should be taken in order to remove systematic errors and ensure the gene expression measurements are comparable across arrays [15]. There is no clear consensus in the community about which methods to use, though several reviews have been published $[8,12]$. After normalisation and statistical tests have been applied, there is an additional problem of multiple testing. Due to the high number of tests taking place (many thousands in most cases), the resulting P-values must be adjusted in order to control or estimate the error rate (see [14] for a review).

The aim of this paper was to summarise and compare the many methods used throughout the EADGENE network (http://www.eadgene.org) for microarray analysis, and compare the results, with the final aim of producing a guide for best practice within the network [4]. This paper describes a variety of methods applied to a simulated data set produced by the SIMAGE package [1]. The data set is a simple comparison of two biological states on ten arrays, with dye-balance. A number of data quality, normalisation and analysis steps were used in various combinations, with differing results.

\subsection{The data}

SIMAGE takes a number of parameters, which were produced using a slide from the real data set as an example [4]. The input values that were used for the current simulations are given in Table I. The simulated data consists of 
ten microarrays each of which represent a direct comparison between different biological samples from situation A and B with a dye balance. SIMAGE assumes a common variance for all genes, something which may not be true for real data. Each slide had 2400 genes in duplicate, with 48 blocks arranged in 12 rows and 4 columns (100 spots per block). Each block was "printed" with a unique print tip. In the simulated data 624 genes were differentially expressed: 264 were up-regulated from A to B while 360 were down regulated. This information was only provided to the participants at the end of the workshop. The simulated data are available upon request from D.J. de Koning (DJ.dekoning@bbsrc.ac.uk).

The data are very noisy with high levels of technical bias and thus provided a serious challenge for the various analysis methods that were applied. Many spots reported background higher than foreground, and others reported a zero foreground signal. Image plots of the arrays showed clear spatial biases in both foreground and background intensities (Fig. 1). Spots, scratches and stripes of background variation are clearly visible, which have been simulated using the "hair" and "disc" parameters of SIMAGE.

All of the slides show a clear relationship between $\mathrm{M}$ (log ratio) and A (average log intensity), and the plots in Figure 2 are exemplars. Slides 3, 5, 6, 7, 9 and 10 displayed a negative relationship between $\mathrm{M}$ and $\mathrm{A}$, whilst the others displayed a positive relationship. Slides 6 and 9 showed an obvious nonlinear relationship between $\mathrm{M}$ and $\mathrm{A}$, but only slide 2 levels off with higher values of A. Finally, Figure 3 shows the range of $M$ values for each array under three different normalisation strategies: none (Fig. 3a), LOESS (Fig. 3b) and LOESS followed by scale normalisation between arrays (Fig. 3c) [17, 19]. It can be seen that before normalisation there is a clear difference in both the median log ratios and the range of log ratios across slides.

This data set was subject to a total of 12 different analysis methods, encompassing a variety of techniques for assessing data quality, normalisation and detecting differential expression. These methods are described in detail and the results of each presented and compared. The results are then discussed in relation to the best methods to use for analysing extremely noisy microarray data.

\section{MATERIALS AND METHODS}

\subsection{Preprocessing and normalisation procedures}

A variety of pre-processing and normalisation procedures were used in combination with the twelve different methods, and these are summarised in 
Table I. Settings for Simage simulation software.

\begin{tabular}{lc}
\hline Array number of grid rows & 12 \\
Array number of grid columns & 4 \\
Number of spots in a grid row & 10 \\
Number of spots in a grid column & 10 \\
Number of spot pins & 48 \\
Number of technical replicates & 2 \\
Number of genes & 0 \\
Number of slides & 10 \\
Perform dye swaps & yes \\
Gene expression filter & yes \\
Reset gene filter for each slide & no \\
Mean signal & 10.33 \\
Change in log ratio due to upregulation & 1.07 \\
Change in log ${ }_{2}$ ratio due to downregulation & -1.26 \\
Variance of gene expression & 2.7 \\
\% of upregulated genes & 15 \\
\% of downregulated genes & 11 \\
Correlation between channels & 1 \\
Dye filter & yes \\
Reset dye filter for each slide & yes \\
Channel variation & 0.2 \\
Gene $\times$ Dye & 0 \\
Error filter & yes \\
Reset error filter for each slide & yes \\
Random noise standard deviation & 0.62 \\
Tail behaviour in the MA plot & 0.108 \\
Non-linearity filter & yes \\
Reset non-linearity filter for each slide & yes \\
Non-linearity parameter curvature & 0.2 \\
Non-linearity parameter tilt & 4.5 \\
Non-linearity from scanner filter & yes \\
Reset non-linearity scanner filter for each slide & yes \\
Scanning device bias & 3 \\
Spotpin deviation filter & 0.04 \\
Reset spotpin filter for each slide & yes \\
Spotpin variation & no \\
Background filter & 0.32 \\
Reset background filter for each slide & yes \\
Number of background densities & yes \\
Mean standard deviation per background density & 5 \\
Maximum of the background signal relative to the non-background signals & 0.2 \\
Standard deviation of the random noise for the background signals & 50 \\
Background gradient filter & 0.1 \\
Reset gradient filter for each slide & no \\
Maximum slope of the linear tilt & yes \\
Missing values filter & 700 \\
Reset missing spots filter for each slide & yes \\
Number of hairs & yes \\
Maximum length of hair & 3 \\
Number of discs & \\
Average radius disc & \\
\hline &
\end{tabular}




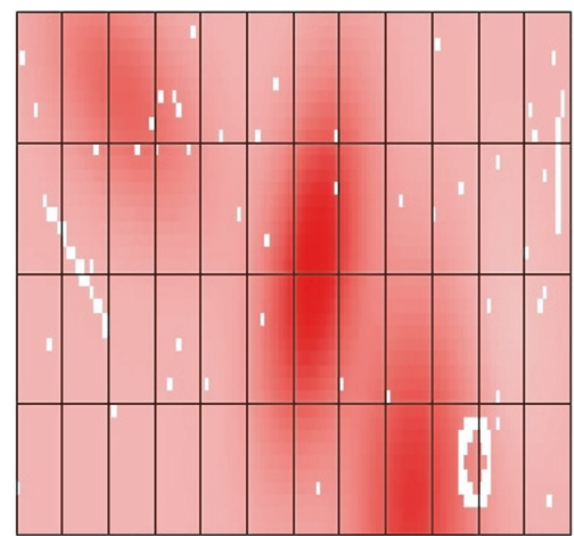

z-range 0 to 643.6 (saturation $0,643.6$ )

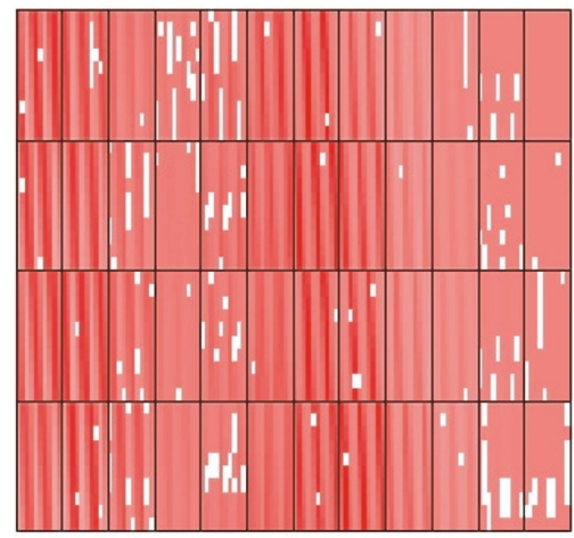

z-range 0 to 643.6 (saturation $0,643.6$ )

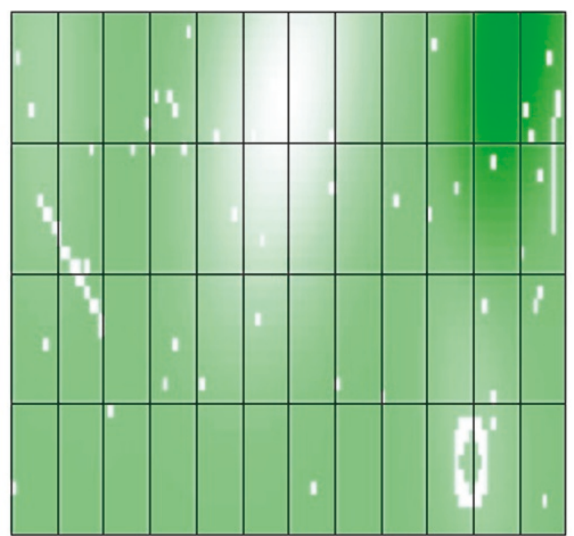

z-range 0 to 458.3 (saturation $0,458.3$ )

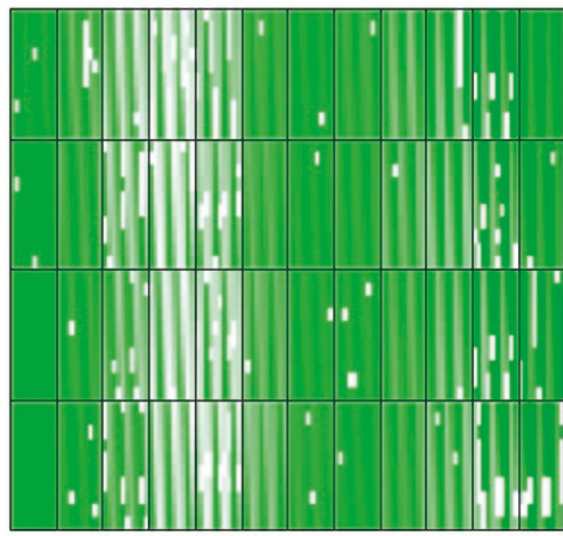

z-range 0 to 470.6 (saturation $0,470.6$ )

Figure 1. Example background plots. The top two images show the background for Cy5 and Cy3 in slide 9, and the bottom two images show the same for slide 10.

Table II. Only one method, IDL1, chose to perform background correction. Some methods chose to eliminate spots, or give them zero weighting, depending on particular data quality statistics; these included having foreground less than a given multiple of background, saturated spots and spots whose intensity was zero. IAH_P1 and IDL1 also removed entire slides considered to have poor data quality. Both IAH_P and IDL submitted two approaches, one based on strict quality control and normalisation, and the second less strict.

Most approaches applied a version of LOWESS or LOESS normalisation, either globally or per print-tip [19]. This is in recognition of the clear relationship between $\mathrm{M}$ and A. Only ROSLIN (assessed normalisation by row and 

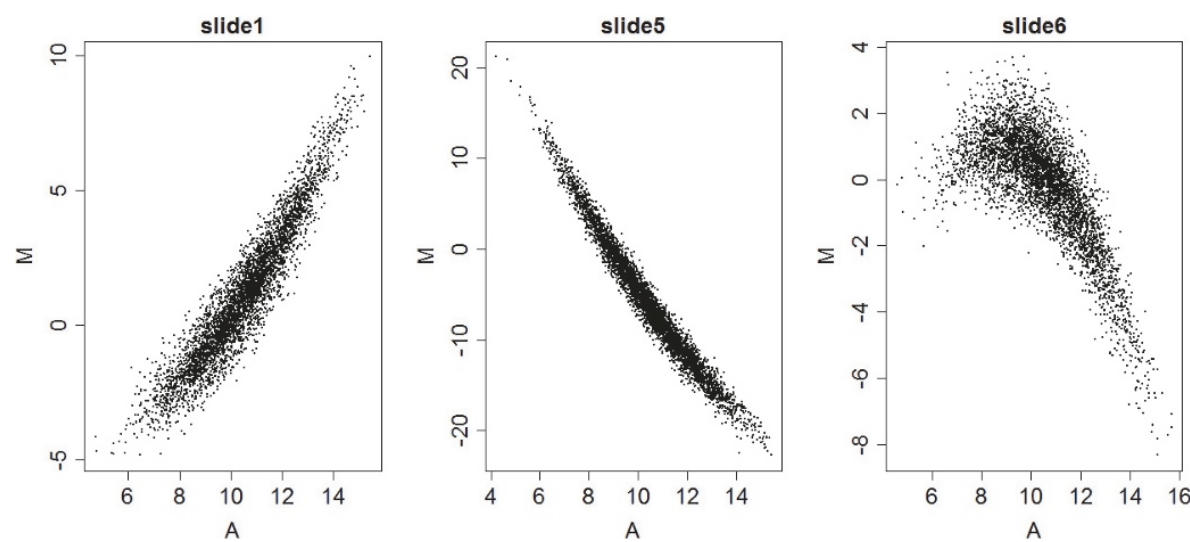

Figure 2. MA-plots of slides 1, 5 and 6. These slides are examples of the three patterns displayed by the simulated data in the MA-space: positive correlation, negative correlation and a more pronounced non-linear correlation.
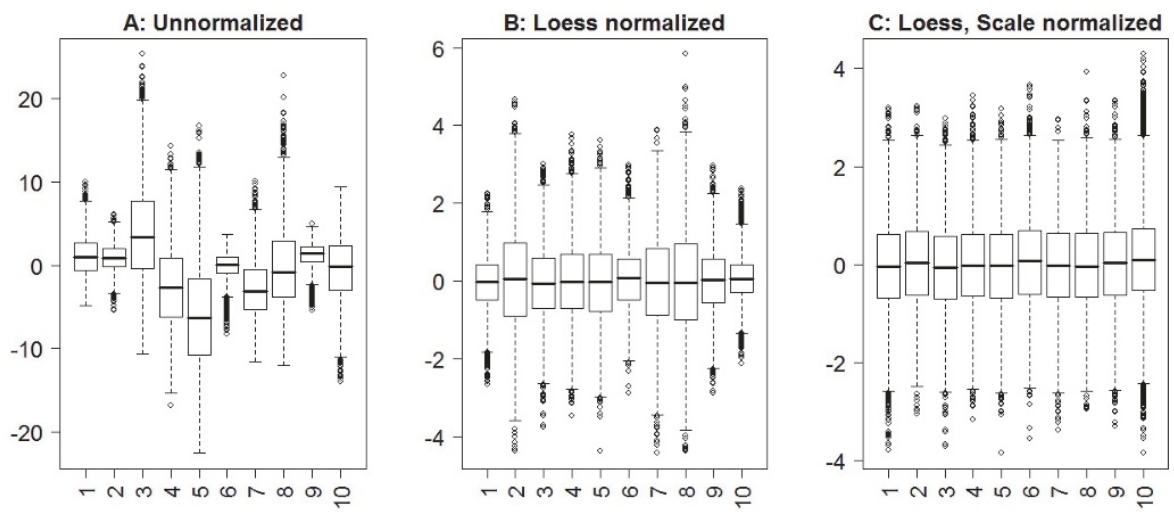

Figure 3. Boxplots of $\mathrm{M}$ values ( $\log _{2}(\mathrm{cy} 5 / \mathrm{cy} 3)$ ) across the 10 arrays for three normalisation strategies: (A) Unnormalised data, (B) LOESS normalised data, and (C) LOESS followed by scale normalised data.

column and found not needed) and INRA_J (correction by block) applied any further spatial normalisation. SLN1 and SLN2 applied median normalisation. Finally, only IDL attempted any correction between arrays by fitting a monotonic spline in MA-space to correct for heterogeneous variance. The smoothing function was fitted to the absolute log ratios (M-values) across the log mean intensities (A-values), and corrected for. This ensured that the variance in $\mathrm{M}$ values was consistent across arrays. 
Table II. Summary of the 12 methods used for analysing the simulated data. "Analysis name" is the name of the analysis method, "Data quality procedures" describe the methods approach to data quality, "Background correction" whether background correction was carried out, "Normalisation" describes the normalisation method and "Differential expression" describes the method's approach to finding differentially expressed genes.

\begin{tabular}{|c|c|c|c|c|}
\hline $\begin{array}{l}\text { Analysis } \\
\text { name }\end{array}$ & $\begin{array}{l}\text { Data quality } \\
\text { procedures }\end{array}$ & $\begin{array}{l}\text { Background } \\
\text { correction }\end{array}$ & Normalisation & $\begin{array}{l}\text { Differential } \\
\text { expression }\end{array}$ \\
\hline$\overline{\text { IAH_P1 }}$ & $\begin{array}{l}\text { Eliminated spots with net } \\
\text { intensity }<0 \text {. } \\
\text { Slides } 5,6 \text { and } 9 \text { deleted }\end{array}$ & No & global LOWESS & $\begin{array}{l}\text { Limma; } \\
\text { FDR correction }\end{array}$ \\
\hline IAH_P2 & Slides 5, 6 and 9 deleted & No & global LOWESS & $\begin{array}{l}\text { Limma; } \\
\text { FDR correction }\end{array}$ \\
\hline \multirow[t]{3}{*}{ IDL1 } & $\begin{array}{l}\text { Eliminated } \\
\text { - control spots } \\
\text { - null spots } \\
\text { - oversaturated spots }\end{array}$ & & & \\
\hline & - values $<3^{*}$ SD bgnd. & & printtip LOWESS; & Limma; \\
\hline & Slides 5 and 7 deleted & Yes & monotonic spline correction & FDR correction \\
\hline IDL2 & & No & $\begin{array}{l}\text { global LOWESS; } \\
\text { monotonic spline correction }\end{array}$ & $\begin{array}{l}\text { Limma; } \\
\text { FDR correction }\end{array}$ \\
\hline INRA_J & Spots $==$ zero removed & No & $\begin{array}{l}\text { LOWESS; } \\
\text { median normalisation by block }\end{array}$ & $\begin{array}{l}\text { structural mixed model; } \\
\text { FDR correction }\end{array}$ \\
\hline INRA_T1 & Spots $==$ zero removed & No & global LOWESS & $\begin{array}{l}\text { Student statistic; } \\
\text { FDR correction }\end{array}$ \\
\hline INRA_T2 & Spots $==$ zero removed & No & global LOWESS & $\begin{array}{l}\text { Student statistic; } \\
\text { Duval correction }\end{array}$ \\
\hline INRA_T3 & Spots $==$ zero removed & No & global LOWESS & $\begin{array}{l}\text { Student statistic; } \\
\text { Bordes correction }\end{array}$ \\
\hline ROSLIN & Spots $==$ zero removed & No & $\begin{array}{l}\text { printtip LOWESS; } \\
\text { row-column normalisation }\end{array}$ & $\begin{array}{l}\text { Limma; } \\
\text { FDR correction }\end{array}$ \\
\hline SLN2 & $\begin{array}{l}\text { Only use data where } \mathrm{FG}> \\
1.5^{*} \mathrm{BG}\end{array}$ & No & median normalisation & Anova (Orange) \\
\hline CDB & $\begin{array}{l}\text { Elimination of spots with } \\
\text { huge M-values }\end{array}$ & No & printtip LOWESS & $\begin{array}{l}\text { fold change cut-off } \\
(+/-0.9)\end{array}$ \\
\hline SLN1 & Excluded BG $>$ FG & No & median normalisation & Anova (GeneSpring) \\
\hline
\end{tabular}

\subsection{Methods for finding differentially expressed genes}

Table II summarises the twelve methods used for analysing the simulated data set. Most variation in the methods came from the area of quality control, with different groups excluding different genes/arrays based on a wide variety of criteria, and correction for multiple testing.

Almost all analysis methods used some variation of linear modelling followed by correction for multiple testing to find differentially expressed genes. The most common of those used was the limma package, which adjusts the t-statistics by empirical Bayes shrinkage of the residual standard errors toward a common value (near to the median) [17]. IAH_P and ROSLIN fitted 
a coefficient for the dye-effect for each gene, which was found to be nonsignificant. IAH_P also adjusted the default estimated proportion of differentially regulated genes in the eBayes procedure to 0.2 once it became clear that a high percentage of the genes in the dataset were differentially regulated. This ensured a good estimate of the posterior probability of differential expression.

Of those that did not use limma, both SLV and SLN2 used an ANOVA approach, implemented in GeneSpring [9] and Orange [5] respectively. INRA_J used a structural mixed model, more completely described in Jaffrézic et al. [11]. CDB employed a cut-off value for the mean log ratio to define the proportion of differentially expressed genes $[10,18]$. INRA_T presented three methods all based on a classic Student statistic and an empirical variance calculated for each gene, but with the P-values adjusted according to Benjamini and Hochberg [2], Duval et al. (partial sums of ordered t-statistics) [6,7] and Bordes et al. (mixture of central and non-central t-statistics) [3]. Apart from INRA_T, those methods that corrected P-values for multiple testing did so using the FDR as described by Benjamini and Hochberg [2]. All corrections for multiple testing were carried out at the $5 \%$ level.

All methods treated the 10 arrays as separate, biological replicates apart from ROSLIN, who treated the dye-swaps as technical replicates. The INRA_J and the three INRA_T methods treated replicate spots as independent measures, resulting in up to 20 values per gene, whereas the other methods averaged over replicate spots. INRA_T reported that preliminary analysis showed very few differences between treating duplicates as independent or by averaging them.

\section{RESULTS}

Table III summarises the results for the analysis of the simulated data set. In terms of the total number of errors made (false positives + false negatives), methods INRA_T2 and INRA_T3 excelled with only 17 and 12 errors respectively. In terms of the least number of false negatives, methods IDL2 and INRA_T1 performed best, having both missed only one gene that was differentially expressed. Many of the analysis methods scored upwards of 95\% correctly identified genes. Of those that did not, IAH_P1 and IDL1 operated strict quality control measures, and may have eliminated a number of differentially expressed genes from the analysis. When the number of correct genes is expressed as a percentage of the number of genes each method identified, these methods too show greater than $95 \%$ correctly identified genes. Those methods based on traditional statistics performed less well than those methods 
Table III. Summary of the results of the analysis of the simulated data set. Table shows the number of genes identified by each method as differentially expressed, the number correct, the number of false positives and negatives, the number of correctly identified genes as a $\%$ of the total number of differentially expressed genes (624) and as a $\%$ of the number of genes identified for each method.

\begin{tabular}{lcccccc}
\hline Analysis & No & Correct & False + & False - & Correct/total & Correct/identified \\
\hline IAH_P1 & 499 & 485 & 14 & 139 & 77.72 & 97.19 \\
IAH_P2 & 608 & 592 & 16 & 32 & 94.87 & 97.37 \\
IDL1 & 304 & 289 & 15 & 335 & 46.31 & 95.07 \\
IDL2 & 642 & 623 & 19 & 1 & 99.84 & 97.04 \\
INRA_J & 663 & 614 & 49 & 10 & 98.40 & 92.61 \\
INRA_T1 & 649 & 623 & 26 & 1 & 99.84 & 95.99 \\
INRA_T2 & 629 & 618 & 11 & 6 & 99.04 & 98.25 \\
INRA_T3 & 622 & 617 & 5 & 7 & 98.88 & 99.20 \\
ROSLIN & 628 & 600 & 28 & 24 & 96.15 & 95.54 \\
SLN2 & 171 & 128 & 43 & 496 & 20.51 & 74.85 \\
CDB & 67 & 44 & 23 & 580 & 7.05 & 65.67 \\
SLN1 & 3 & 3 & 0 & 621 & 0.48 & 100.00 \\
\hline
\end{tabular}

specifically designed with microarray data in mind. CDB chose a fold-change cut-off above which genes were flagged as significant, set at a $\log _{2}$ ratio of $+/-0.9$. SLN1 analysed the dye-swap slides separately, which will have reduced the statistical power of the analysis, combining the results afterwards. This resulted in only three genes identified as differentially expressed; however, all were correct. SLN2 identified 171 genes as differentially expressed, but also showed a relatively high number of false positives and negatives.

Table IV shows the top ten differentially expressed genes that were missed by the 12 methods (false negatives). One gene, gene 203, was missed by every analysis method. Genes 2221 and 465 were missed by all but two methods, those being IDL2 and INRA_T1 in both cases. These genes are characterised by $\log$ ratios that do not necessarily match their direction of regulation and very large standard deviations relative to the normalised mean log ratios.

Table $\mathrm{V}$ shows the top ten genes wrongly identified as differentially expressed by the 12 analysis methods (false positives). Gene 1819 was identified as differentially expressed in 8 of the 12 methods; however, given that CDB, SLN1 and SLN2 identified very few genes in total, this means that only one of the more accurate methods correctly called this gene as not differentially expressed, and that is INRA_T3. Moving further down, there are four genes called as false positives in six of the methods, though there is no consistency 
Table IV. The top ten genes identified as false negatives in the 12 analysis methods. Table contains the gene id (gene), mean and standard deviation of the unnormalised $\log$ ratio (M and SD), mean and standard deviation of the LOESS normalised log ratio (M LOESS and SD LOESS), the number of methods in which the gene was a false negative (Count) and the direction of regulation from SIMAGE (Regulated).

\begin{tabular}{lcccccc}
\hline Gene & M & SD & M LOESS & SD LOESS & Count & Regulated \\
\hline gene203 & -1.35 & 3.25 & -0.01 & 0.65 & 12 & up \\
gene2221 & -1.71 & 3.14 & -0.40 & 0.39 & 10 & up \\
gene465 & -0.70 & 3.00 & -0.39 & 0.59 & 10 & up \\
gene1411 & 2.74 & 6.80 & -0.48 & 0.67 & 9 & up \\
gene352 & 0.63 & 3.97 & -0.39 & 0.84 & 8 & up \\
gene1448 & -4.24 & 6.26 & -1.32 & 1.87 & 7 & down \\
gene1580 & -2.12 & 3.58 & -0.58 & 0.89 & 7 & up \\
gene1667 & 2.59 & 6.61 & 0.69 & 0.78 & 7 & up \\
gene1704 & -2.26 & 4.16 & -0.46 & 1.11 & 7 & up \\
gene90 & 3.06 & 6.53 & -0.47 & 1.01 & 7 & up \\
\hline
\end{tabular}

Table V. The top ten genes identified as false positives in the 12 analysis methods. The table contains the gene id (gene), mean and standard deviation of the unnormalised $\log$ ratio (M and SD), mean and standard deviation of the LOESS normalised log ratio (M LOESS and SD LOESS) and the number of methods in which the gene was a false positive (Count).

\begin{tabular}{lrcccc}
\hline Gene & M & SD & M LOESS & SD LOESS & Count \\
\hline gene1819 & 1.93 & 4.67 & 0.50 & 0.42 & 8 \\
gene2262 & -0.65 & 3.45 & 0.65 & 0.67 & 6 \\
gene555 & 0.72 & 3.75 & -0.55 & 0.65 & 6 \\
gene995 & 0.18 & 2.93 & 0.60 & 0.65 & 6 \\
gene999 & -0.18 & 3.30 & 0.54 & 0.38 & 6 \\
gene1258 & 1.98 & 5.04 & 0.48 & 0.52 & 5 \\
gene1324 & -0.12 & 3.34 & 0.60 & 0.44 & 5 \\
gene1654 & 0.33 & 3.69 & 0.52 & 0.61 & 4 \\
gene2069 & -0.35 & 4.04 & -0.33 & 0.51 & 4 \\
gene2110 & 3.40 & 5.07 & 0.49 & 0.61 & 4 \\
\hline
\end{tabular}


shown in which methods identified those four correctly or incorrectly. These genes are characterised by standard deviations that are about equal to the normalised log ratios, in contrast to the false negatives.

\section{DISCUSSION}

After the comparison, we are in the unique position of knowing a priori which and how many genes were differentially expressed, however before starting the analysis none of the groups had the information and only a very noisy data set was provided. Each group applied a different variety of techniques to find the differentially expressed genes. In some cases, the data were put into a standardised pipeline, and in others the analysis was customised to this data set.

It is interesting to note that only one method used any kind of background subtraction. This was due to researchers recognising that although some slides displayed high background, there was little relationship with spot foreground, and therefore subtracting background would have removed many spots from the analysis with no resulting benefit. A consensus in the wider community on background correction has yet to be reached, however the partners within the EADGENE network appeared to have done so, with all but one partner deciding not to correct for local background when analysing this data set.

Applying stringent spot quality procedures and subsequent elimination of both spots and slides from the analysis, as seen in IAH_P1 and IDL1, did not greatly lessen the number of false positives, but greatly increased the number of false negatives. The increase in false negatives was much larger than the corresponding decrease in false positives. This suggests that, when dealing with noisy data, care must be taken to eliminate only data for which a real physical source of error can be identified, e.g. detector saturation during scanning. In the case of the data analysed here some of the simulated backgrounds were high, leading some groups to reject those spots; in fact, rejecting the estimated backgrounds was the best approach, since eliminating data from the analysis leads to the elimination of significantly differentially expressed genes with no associated benefit.

It is clear from the relationship between $\mathrm{M}$ and $\mathrm{A}$ that an intensity dependent normalisation should be used on these data and most groups reflected that by choosing to use LOWESS/LOESS normalisation. The spatial biases shown in the background suggest that perhaps a spatial normalisation technique should be used, yet only two investigated the need for it: INRA_J and ROSLIN. The differences seen in the range of raw log ratios between slides 
suggest that a between-slides normalisation method would have been appropriate, yet only IDL attempted to do so. Figure 3 shows the range of $\mathrm{M}$ values for each array under three different normalisation strategies: none (Fig. 3a), LOESS (Fig. 3b) and LOESS followed by scale normalisation between arrays (Fig. 3c) $[17,19]$. Figure 3a shows that there is a large amount of variation in the range of $\mathrm{M}$ values between slides, and Figure $3 \mathrm{~b}$ shows that that variation is not entirely removed by LOESS normalisation alone. Figure $3 \mathrm{c}$ shows the most uniform distribution of $\mathrm{M}$ values across arrays, as can be expected given the normalisation strategy. Whether or not this is desirable depends on the context of the experiment. For example, one would expect technical replicates to have very similar distributions, whereas biological replicates may not. In this experiment, if we assume that the dye-swapped arrays are technical replicates, then array pairs 5 and 6, and 9 and 10, represent technical replicates of one another, yet show vastly differing ranges of $\mathrm{M}$ values (Fig. 3a), adding weight to the argument for between array normalisation. The failure to apply additional normalisation steps after the first may have been due to fears of "over-fitting" the data. However, ROSLIN report that additional analyses were carried out on the data with between-slides variation-standardisation applied, and an additional 23 genes were identified, 12 of which were differentially expressed, the other 11 being false positives (data not shown).

The approaches may be split into traditional and more sophisticated methods of analysis. SLN1, SLN2 and CDB employed more traditional methods (analysis of variance and fold-change cut-off), whereas the others employed methods shown to be of particular use with microarray data. The authors from CDB wish it to be known that theirs was only a preliminary analysis. DNMAD [18] and GEPAS [10] are sophisticated tools for the analysis of microarray data, and it is unfortunate that some of their more sophisticated methods were not brought to bear on the simulated data. The more traditional methods were also more conservative, identifying fewer genes in total as differentially regulated. They did not, however, have correspondingly smaller false positive rates.

Examination of the genes consistently appearing as false negatives or false positives reveals predictable trends. Consistent false negatives showed very high variation about the mean, whereas consistent false positives showed much less. The simulation software, SIMAGE, gives the same ratio to all genes designated up- or down-regulated, therefore any difference between genes designated as up- or down-regulated is solely down to noise modelled by the software. Those genes consistently identified as false negatives simply received more noise, and those consistently identified as false positives received less. 
Overall, given that this was a noisy data set, it is promising that such high numbers of correctly identified genes can be achieved. The trade off between false positives and false negatives can clearly be seen and suggests that elimination of data due to poor spot quality measures does not pay off in terms of the decrease in false positives given the large increase in false negatives. Correction for the false discovery rate (FDR) [2] was the most commonly used technique for adjusting P-values. However, a direct comparison of multiple testing procedures occurred in the INRA_T analyses, with the two novel methods presented out-performing the FDR procedure proposed by Benjamini and Hochberg [2] in terms of error rate; the mixture model described by Bordes et al. [3] performed particularly well. The performance of the INRA_T methods is of note given that similar gene-by-gene methods have been shown to lack power in comparison to shrinkage methods such as limma [17] and the structural model [11]. It may be that the data was sufficiently well replicated to overcome this. In addition, this data set has been simulated with homogeneous variances, and this assumption may not hold true for real data sets.

It should be noted that the simulated data represents a well replicated experiment, with ten replicates for a single comparison. This no doubt lends a great deal of power to the analyses. Additional power was achieved by INRA_J and the three INRA_T methods by treating replicate spots as independent measures, resulting in up to twenty measurements per gene. Although these four techniques showed very good results, comparable results were achieved by ROSLIN, IAH_P2 and IDL2, showing that the increase in replication from ten to twenty did not greatly improve the results. In fact, the IAH_P2 analysis, which eliminated 3 out of the 10 slides but still achieved very high success rates, showed that this data set was probably over-endowed with replicates, beyond what would normally be found in a real experiment. Repeating the analyses with a smaller number of replicates may be informative. Kooperberg et al. [13] compared methods for analysing microarray experiments with small numbers of replicates and concluded that the best methods were those which took an empirical Bayes approach (e.g. [17], used in some analyses presented here) and those that combined similar experiments.

\section{ACKNOWLEDGEMENTS}

The authors acknowledge the Danish participants and WP1.4 for organising the workshop and EADGENE for financial support (EU Contract No. FOODCT-2004-506416). 


\section{REFERENCES}

[1] Albers C.J., Jansen R.C., Kok J., Kuipers O.P., van Hijum S.A., SIMAGE: simulation of DNA-microarray gene expression data, BMC Bioinformatics 7 (2006) 205.

[2] Benjamini Y., Hochberg Y., Controlling the false discovery rate: a practical and powerful approach to multiple testing, J. Royal Stat. Soc. Ser. B 57 (1995) 289300.

[3] Bordes L., Delmas C., Vandekerkhove P., Semiparametric estimation of a two component mixture model when a component is known, Scand. J. Stat. 33 (2006) $733-752$.

[4] de Koning D.J., Jaffrézic F., Lund M.S., Watson M., Channing C., Hulsegge I., Pool M.H., Buitenhuis B., Hedegaard J., Hornshøj H., Jiang L., Sørensen P., Marot G., Delmas C., Lê Cao K.-A., San Cristobal M., Baron M.D., Malinverni R., Stella A., Brunner R.M., Seyfert H.-M., Jensen K., Mouzaki D., Waddington D., Jiménez-Marín Á., Pérez-Alegre M., Pérez-Reinado E., Closset R., Detilleux J.C., Dovč P., Lavrič M., Nie H., Janss L., The EADGENE microarray data analysis workshop, Genet. Sel. Evol. 39 (2007) 621-631.

[5] Demsar J., Zupan B., Leban G., Orange: From Experimental Machine Learning to Interactive Data Mining, White Paper (http://ww.ailab.si/orange) (2004), Faculty of Computer and Information Science, University of Ljubljana.

[6] Duval M., Degrelle S., Delmas C., Hue I., Laurent B., Robert-Granié C., A novel procedure to determine differentially expressed genes between two conditions, 8th World Congress on Genetics Applied to Livestock Production, Belo Horizonte (Brazil), August 13-18, 2006.

[7] Duval M., Delmas C., Laurent B., Robert-Granié C., A simple procedure to detect noncentral observations from a sample, http://www.lsp.upstlse.fr/Recherche/Publications/2006/duv06.html.

[8] Fujita A., Sato J.R., Rodrigues L. de O., Ferreira C.E., Sogayar M.C., Evaluating different methods of microarray data normalization, BMC Bioinformatics 7 (2006) 469.

[9] GeneSpring GX, http:// www.agilent.com/chem/genespring.

[10] Herrero J., Al-Shahrour F., Díaz-Uriarte R., Mateos A., Vaquerizas J.M., Santoyo J., Dopazo J., GEPAS: A web-based resource for microarray gene expression data analysis, Nucleic Acids Res. 31 (2003) 3461-3467.

[11] Jaffrézic F., Marot G., Degrelle S., Hue I., Foulley J.L., A structural mixed model for variances in differential gene expression studies, Genet. Res. 89 (2007) 1925.

[12] Jeffery I.B., Higgins D.G., Culhane A.C., Comparison and evaluation of methods for generating differentially expressed gene lists from microarray data, BMC Bioinformatics 7 (2006) 359.

[13] Kooperberg C., Aragaki A., Strand A.D., Olson J.M., Significance testing for small microarray experiments, Stat. Med. 24 (15) (2005) 2281-2298.

[14] Pounds S.B., Estimation and control of multiple testing error rates for microarray studies, Brief. Bioinform. 7 (2006) 25-36. 
[15] Quackenbush J., Microarray data normalization and transformation, Nat. Genet. 32 (2002) 496-501.

[16] Schena M., Shalon D., Davis R.W., Brown P.O., Quantitative monitoring of gene expression patterns with a complementary DNA microarray, Science 270 (1995) 467-470.

[17] Smyth G.K., Linear models and empirical Bayes methods for assessing differential expression in microarray experiments, Stat. Appl. Genet. Mol. Biol. 3 (2002) Article 3.

[18] Vaquerizas J.M., Dopazo J., Díaz-Uriarte R., DNMAD: web-based diagnosis and normalization for microarray data, Bioinformatics 20 (2002) 3656-3658.

[19] Yang Y.H., Dudoit S., Luu P., Lin D.M., Peng V., Ngai J., Speed T.P., Normalization for cDNA microarray data: a robust composite method addressing single and multiple slide systematic variation, Nucleic Acids Res. 30 (2002) e15. 